\title{
Identification of anthracnose (Colletotrichum lentis) race 1 resistance loci in lentil by integrating linkage mapping and a genome-wide association study
}

\author{
Tadesse S. Gela, Larissa Ramsay, Teketel A. Haile, Albert Vandenberg, and Kirstin E. Bett* \\ Crop Development Centre, Department of Plant Sciences, University of Saskatchewan, 51 \\ Campus Drive, Saskatoon, Saskatchewan, S7N 5A8, Canada. \\ *E-mail corresponding author: k.bett@usask.ca
}

\begin{abstract}
Anthracnose, caused by Colletotrichum lentis, is a devastating disease of lentil in Western Canada. Growing resistant lentil cultivars is the most cost-effective and environmentally friendly approach to prevent seed yield losses that can exceed $70 \%$. To identify loci conferring resistance to anthracnose race 1 in lentil, biparental quantitative trait loci (QTL) mapping of two recombinant inbred line (RIL) populations was integrated with a genome-wide association study (GWAS) using 200 diverse lentil accessions from a lentil diversity panel (LDP). A major-effect QTL (qAnt1.Lc3) conferring resistance to race 1 was mapped to lentil chromosome 3 and co-located on the lentil physical map for both RIL populations. Clusters of candidate nucleotide binding-leucine-rich repeats (NB-LRR) and other defense-related genes were uncovered within the QTL region. A GWAS detected 14 significant SNP markers associated with race 1 resistance on chromosomes 3, 4, 5, and 6. The most significant GWAS SNPs on chromosome 3 supported qAnt1.Lc-3 and delineated a region of 1.6 Mb containing candidate resistance genes. The identified SNP markers can be directly applied in marker-assisted selection to accelerate the introgression of race 1 resistance in lentil breeding.
\end{abstract}

Keywords: Cultivated lentil, Disease resistance, GWAS, QTL mapping, Resistance genes 


\section{INTRODUCTION}

Lentil (Lens culinaris Medik., $2 \mathrm{n}=2 \mathrm{x}=14$ ) is an annual self-pollinating pulse crop with a genome size of $\sim 4 \mathrm{~Gb}$ (Arumuganathan and Earle, 1991). The crop is cultivated in more than 70 countries worldwide, with the production from Canada providing $40 \%$ of the world's supply in recent years (2013-2019 average, FAOSTAT, 2021). Lentil provides an affordable source of dietary proteins, minerals, fiber, and carbohydrates and plays a vital role in alleviating malnutrition and micronutrient deficiencies (Srivastava and Vasishtha, 2012). Lentil production in the northern Great Plains of North America, particularly western Canada, is challenged by anthracnose caused by the fungus Colletotrichum lentis (Damm) (Damm et al., 2014). Yield loss of more than 70\% can occur when susceptible cultivars experience high disease pressure (Chongo et al., 1999; Morrall and Pedersen, 1991). The disease has also been reported in Bangladesh, Bulgaria, Brazil, Ethiopia, Morocco, Pakistan, Syria, and USA and is considered of minor importance in other parts of the world (Bellar and Kebabeh, 1983; Bayaa and Erskine, 1997; Morrall, 1997; Kaiser et al., 1998).

Breeding and deployment of resistant cultivars is an important component of an integrated disease management strategy for preventing yield loss caused by anthracnose. Successful deployment requires continuous incorporation of new sources of resistance into elite breeding materials. Two pathogenic races of $C$. lentis (race 0 and race 1) have been described (Buchwaldt et al., 2004; Banniza et al., 2018). In cultivated lentil, no sources of high levels of resistance to the highly virulent race 0 have been found, however, resistance does occur in Lens ervoides in the tertiary gene pool (Tullu et al., 2006). Lentil accessions with resistance to anthracnose race 1 were identified in L. culinaris germplasm (Buchwaldt et al., 2004). Resistance to race 1 was transferred 
into elite breeding lines and resulted in the release of cultivars such as 'CDC Robin' and 'CDC Redberry' (Vandenberg et al., 2002, 2006). Since then, several lentil cultivars with partial resistance to race 1 have been released and deployed for production in Saskatchewan (Government of Saskatchewan, 2019).

Successful incorporation of race 1 resistance into improved lentil cultivars is possible through classical breeding but could be greatly improved if molecular breeding approaches could be employed. This requires use of molecular markers to identify the genes that control the quantitative traits; however, little is known about the causal genomic regions controlling race 1 resistance in lentil. Tullu et al. (2003) mapped race 1 resistance in lentil accession PI 320937 using RAPD markers and identified a major dominant gene and several minor genes. Segregation analysis of race 1 resistance in PI 320952 and PI 345629 revealed control by recessive and dominant genes (Buchwaldt et al., 2013).

More precise knowledge of accurate localization of QTL/genes and identification of linked molecular markers is an important step in development of effective MAS in lentil breeding. It also facilitates pyramiding of the resistance genes into lentil cultivars to achieve high levels of resistance against both races of anthracnose. Integration of QTL mapping in biparental populations and GWAS provides the technology to identify trait loci associated with resistance while refining the genomic regions with high resolution (Zhu et al., 2008). Both mapping strategies have been successfully used to identify QTL for multiple traits in lentil (biparental: Fedoruk et al., 2013, Ates et al., 2016; Aldemir et al., 2017; Subedi et al., 2018; Sari et al., 2018; Polanco et al., 2019; and GWAS: Khazaei et al., 2018; Kumar et al., 2018; Kumar et al., 2019). 
In lentil, the low number and density of markers used for mapping studies have been the limiting factor for a long time (reviewed by Kumar et al., 2015). However, advances in next generation sequencing technologies have enabled the detection of large numbers of SNP sets from different marker genotyping platforms in lentil. Exome capture genotyping, which targets the genic regions of the genome, has been demonstrated to be an efficient method of high-throughput SNP discovery in lentil (Ogutcen et al., 2018). The SNP markers targeting the functional region of a genome may be of great importance to breeders because they are attributable to traits of interest under artificial selection through MAS. Here, we used exome capture genotyping on a diversity panel of lentil accessions and a lentil biparental RIL population (Haile et al., 2020). The objective of this research were: (i) to identify QTL for anthracnose race 1 resistance in two lentil RIL populations, (ii) to detect chromosomal regions associated with race 1 resistance using GWAS, and (iii) to compare the genomic regions detected in both mapping strategies to identify candidate genes involved in disease resistance.

\section{MATERIAL AND METHODS}

\section{Plant materials}

Two biparental-derived lentil RIL populations (LR-01 and LR-18) and a GWAS panel were used to identify loci conferring resistance to anthracnose race 1, LR-01 was derived from the cross ILL $1704 \times$ CDC Robin (Haile et al., 2020); LR-18 was developed from the cross CDC Robin $\times$ 964a46 (Tar'an et al., 2003). Both RIL populations were advanced to $\mathrm{F}_{7}$ by single seed descent before bulking and comprised a set of 102 and 139 RILs for LR-01 and LR-18, respectively. CDC Robin is a cultivar partially resistant to anthracnose race 1 and resistant to ascochyta blight (Vandenberg

et al., 2002). Parents ILL 1704 and 964a-46 are susceptible to anthracnose race 1 . The pedigree of 
breeding line 964a-46 includes ILL 5588, an ascochyta blight resistant landrace released as the cultivar Northfield in Australia (Ali, 1995). ILL 1704 is a landrace from Ethiopia with moderate resistance to ascochyta blight (Tullu et al., 2010). The GWAS panel was a subset of 200 lentil genotypes selected from the Lentil Diversity Panel (LDP; N=324) (Haile et al., 2020; Wright et al., 2021; http://knowpulse.usask.ca/Lentil-Diversity-Panel). The LDP consists of 324 accessions assembled from the gene banks of Plant Gene Resources of Canada (PGRC), the USDA, the International Center for Agricultural Research in the Dry Areas (ICARDA), and includes cultivars developed at the Crop Development Centre (CDC), University of Saskatchewan (USask).

\section{Disease phenotyping}

Colletotrichum lentis isolate CT-21 representing race 1 (Banniza et al., 2018) was used to inoculate the RIL populations and GWAS panel. Fungal inoculum production and inoculation procedures were done as described by Gela et al. (2020). Disease reactions of the RIL populations and parents were evaluated in a growth chamber (GR48, Conviron, Winnipeg, Canada) environment at the USask College of Agriculture and Bioresources phytotron facility, Saskatoon, Canada. The GWAS panel was evaluated in a growth chamber and in an outdoor polyhouse. In the growth chambers evaluations, plants of each accession/RIL were grown in replicates, each consisting of sets of 38cell cone trays $(26.8 \mathrm{~cm} \times 53.5 \mathrm{~cm})$ per replication. The trays were filled with Sun Gro Horticulture Sunshine Mix LA4 (Sun Gro Horticulture, Bellevue, USA) and perlite (Specialty Vermiculite Canada, Winnipeg, MB) at 3:1 ratio (v/v). The susceptible control cultivar 'Eston' (Slinkard, 1981) and the RIL parental genotypes were included in each tray. Four weeks after seeding, plants were inoculated with a spore suspension $\left(5 \times 10^{4}\right.$ spores $\left.\mathrm{mL}^{-1}\right)$ at $3 \mathrm{~mL}$ per plant using an airbrush. Plants were placed in an incubation chamber (relative humidity 90-100\%) for $48 \mathrm{~h}$ before being 
moved to misting benches (see Gela et al., 2020). The experiments were conducted separately for each population and arranged in randomized complete block design with five and seven replications for the RIL populations and the GWAS panel, respectively, which were blocked over time. For the RIL populations, two plants of each RIL were included in each of five sequential experimental runs and the mean disease severity score of the two plants per replicate was used for statistical analysis. For the GWAS panel, one plant of each accession was evaluated as an individual and repeated seven times. For GWAS accessions exhibiting segregation for disease reaction, an additional three runs were conducted to obtain representative disease scores. For all experiments the plants were scored for race 1 disease severity at 8-10 days post-inoculation (dpi) using a 0 to 10 rating scale with $10 \%$ increments.

The polyhouse experiment was conducted at the Department of Plant Sciences field laboratory at the USask. Four seeds of each accession and two seeds of Eston (susceptible control) were sown in individual $4.5 \mathrm{~L}$ pots $(15.5 \mathrm{~cm}$ diameter) containing Sunshine Mix No. 4 (Sun Grow Horticulture ${ }^{\circledR}$ Ltd., Vancouver, BC, Canada). The plants were grown under open field conditions for 6 weeks (early flowering stage), then immediately before inoculation, polyhouse tunnel covers of translucent thin plastic sheeting were installed by suspending them $1.5 \mathrm{~m}$ above the ground over the pots. The area under the cover was equipped with a misting irrigation system. Each pot was sprayed with approximately $36 \mathrm{~mL}\left(6 \mathrm{~mL}\right.$ plant $\left.{ }^{-1}\right)$ of aqueous spore suspension $\left(5 \times 10^{4}\right.$ spores $\mathrm{mL}^{-1}$ ) of race 1 isolate CT-21 using a pressurized knapsack sprayer. The inoculations were performed in the evening to avoid high temperature conditions and to facilitate the germination of spores on the leaves. To promote disease development after inoculation, misting irrigation was applied from early morning to evening for $30 \mathrm{~s}$ every $15 \mathrm{~min}$. The experiment was conducted in a 
randomized complete block design (RCBD) with three replications. Disease severity data were collected $14 \mathrm{~d}$ after inoculation using a 0 to 10 rating scale with $10 \%$ increments. Data were converted to percentage disease severity using the class midpoints for data analysis.

\section{Statistical analysis of phenotypic data}

Analysis of variance (ANOVA) for the phenotypic data were performed using the PROC GLIMMIX procedure of SAS v.9.4 (SAS Institute, Cary, USA). Broad-sense heritability $\left(\mathrm{h}_{\mathrm{B}}{ }^{2}\right)$ of single and combined environments disease severity scores of the GWAS panel were calculated with the lme4 package (Bates et al., 2015) in $\mathrm{R}$ (R Core Team, 2020) using the equation: $\sigma^{2}{ }_{\mathrm{G}} /\left(\sigma_{\mathrm{G}}^{2}+\sigma^{2} \mathrm{e} / \mathrm{r}\right)$ and $\sigma_{\mathrm{G}}^{2} /\left[\left(\sigma_{\mathrm{G}}^{2}\right)+\left(\sigma_{\mathrm{GE}}^{2} / \mathrm{E}\right)+\left(\sigma^{2} \mathrm{e} / \mathrm{Er}\right)\right]$, where $\sigma_{\mathrm{G}}^{2}$ is the genotypic variance, $\sigma_{\mathrm{GE}}^{2}$ is variance of the genotype $\times$ environment interactions, $\sigma^{2} \mathrm{e}$ is the error variance, $\mathrm{r}$ is the number of replications in each environment and $\mathrm{E}$ is the number of environments (Knapp et al., 1985). Lsmeans of disease severity scores were calculated for each environment and for combined environments and subjected to square root transformation to improve the normality of the skewed distribution to perform GWAS analysis as described by Li et al. (2019). Spearman's rank correlation of disease severity between test environments was performed using the procedure CORR in SAS. For RIL populations, mean disease severity data were calculated from the replicates and used for QTL mapping.

\section{SNP genotyping}

Genomic DNA was isolated from fresh leaves of 2 to 3 -week-old seedlings of the GWAS panel and LR-01 populations, including the parents (Haile et al., 2020). All lines were genotyped with a custom exome capture assay using protocols previously described by Ogutcen et al. (2018). The 
SNPs were called using lentil reference genome (CDC Redberry genome assemble v2.0; Ramsay et al., 2019). The resulting variant call format (VCF) file was filtered using VCFtools (Danecek et al., 2011) to retain SNPs with less than 10\% missing allele calls (minimum read depth=5) and SNPs with minor allele frequency greater than $5 \%$.

\section{Linkage map construction and QTL mapping}

LR-01 population. A draft genetic linkage map consisting of 21,634 SNPs grouped into seven linkage groups corresponding to the seven haploid lentil chromosomes was generated using the MSTMap software (Wu et al., 2008). This high-density genetic map was further subjected to bin grouping of the redundant SNP markers using BIN functionality employed in QTL ICIMapping 4.1 software (Meng et al., 2015). A marker representing each bin was retained on the map, and the map distances in centimorgans $(\mathrm{cM})$ between markers were calculated using the Kosambi function (Kosambi, 1944). All genotyping information for the LR-01 genetic linkage map can be found through the KnowPulse database accessible at: https://knowpulse.usask.ca/Geneticmap/2695342 (accessed January 6, 2021).

LR-18 population. The genetic linkage map developed earlier by Fedoruk et al. (2013) using the SNPs assayed using a 1536-SNP Illumina Golden Gate array (Illumina, San Diego, CA) was used for QTL mapping. The map consisted of 550 SNP markers, seven SSR markers, and four morphological markers. The total map distance was $697 \mathrm{cM}$ with an average marker distance of $1.2 \mathrm{cM}$. All genotyping information for the LR-18 genetic linkage map can be found through the KnowPulse database accessible at: https://knowpulse.usask.ca/Geneticmap/2686922 (accessed January 13, 2021). 
The QTL analyses were performed using R/qtl software (http://www.rqtl.org/; Broman et al., 2003). The QTL genotype probabilities were calculated along the chromosome at $1 \mathrm{cM}$ intervals assuming a genotyping error rate of $1.0 \mathrm{e}^{-4}$ and using the Kosambi map function (Kosambi 1944). Multiple QTL mapping was completed with the stepwiseqtl function (Broman et al., 2003) using Haley-Knott regression (Haley and Knott, 1992). The optimal QTL model was chosen based on the highest penalized LOD score (Manichaikul et al., 2009) after forward and backward selection and elimination modelling using stepwiseqt function. Penalties for model selection and genomewide significance threshold $(\alpha=0.05)$ were determined by 1000 permutations with scantwo function for two-dimensional QTL scan. The confidence intervals for each QTL were estimated using the "lodint" function that calculated the 1.5 LOD support intervals. The percentage of the phenotypic variance explained (PVE) and effects of QTLs were obtained by fitting a mixed linear model using the "fitqtl" function (Broman et al., 2003).

\section{Genome-wide association analysis}

The population structure of the association mapping panel was assessed using a pruned subset of 6,516 unlinked SNP markers generated after removing SNPs with minor allele frequency of $<10 \%$ and linkage disequilibrium $\left(\mathrm{r}^{2}<0.2\right)$ at a sliding window of $1 \mathrm{Mb}$ using SNPRelate package (Zheng et al., 2012). The Bayesian model-based clustering implemented in STRUCTURE V2.3.4 (Pritchard et al., 2000) was used to estimate the number of subpopulations (K). The number of K sets from $K=2$ to $K=10$, with 10 times independent runs for each $K, 50,000$ burn-in iterations, and 100,000 Markov chain Monte Carlo sampling replicates were conducted. The optimal K-values were determined using STRUCTURE HARVESTER (Earl \& vonHoldt, 2012), and visualized by STRUCTURE PLOT (Ramasamy et al., 2014). Genotypes with membership probabilities $<60 \%$ 
were considered admixtures (Falush et al., 2003). Principal component analysis (PCA) (Patterson et al., 2006) and the genetic kinship matrix were conducted using the Genomic Association and Prediction Integrated Tool (GAPIT) (Lipka et al., 2012).

Marker-trait associations (MTA) were tested using a compressed mixed linear model (CMLM) (Zhang et al., 2010) including the population structure (Q) and kinship (K). Association tests were run using the software GAPIT implemented in R software (Lipka et al., 2012). The quantilequantile $(\mathrm{Q}-\mathrm{Q})$ plot's fattiness was inspected to compare the results from $\mathrm{Q}+\mathrm{K}$ (population structure and kinship), and PC $+\mathrm{K}$ (principal component and kinship) analysis. However, because the Q-Q plots generated using the two approaches were similar, only the results of the PC+K analysis are presented (Supplemental Figure S3). SNPs with - $\log _{10}(\mathrm{p}$-value) $\geq 5.2$ were considered to have significant associations based on a Bonferroni threshold $(1 / \mathrm{n})$ correction at $\mathrm{p}>6.6 \times 10^{-6}$. Manhattan and Q-Q plots were generated with the R package qqman (Turner, 2014).

\section{Candidate gene analysis}

The physical location of markers that defined the QTL intervals were identified using the CDC Redberry genome assembly v.2.0 (Ramsay et al., 2019) and examined for the presence of candidate genes associated with disease resistance. The annotated genes identified were used in BLAST analysis of GenBank (NCBI) database to confirm their functions in other plant species. All reported disease resistance (R-) or defense-related genes in plants were considered for identification of candidate genes. 


\section{RESULTS}

\section{Phenotypic variation}

In both RIL populations, the resistant parent CDC Robin had a resistant reaction with mean disease severity of $23 \%$. The susceptible parents, ILL 1704 and $964 a-46$ had susceptible reactions with the mean disease scores of 95\% and 93\%, respectively. Most of the RILs exhibited disease severity between the range of their parental lines, but some even exhibited lower disease severity than CDC Robin (Figure $1 \mathrm{a}$ and $\mathrm{b}$ ). The disease reactions of the two RIL populations to anthracnose race 1 ranged from 5 to $95 \%$, with an overall average mean of $53.6 \%$ for LR-01 and $54.2 \%$ for LR-18. The RILs in both populations had bimodal frequency distributions which fitted a 1:1 resistant to susceptible segregation ratio, indicating monogenic segregation for resistance to anthracnose race 1 (Supplemental Table S1).
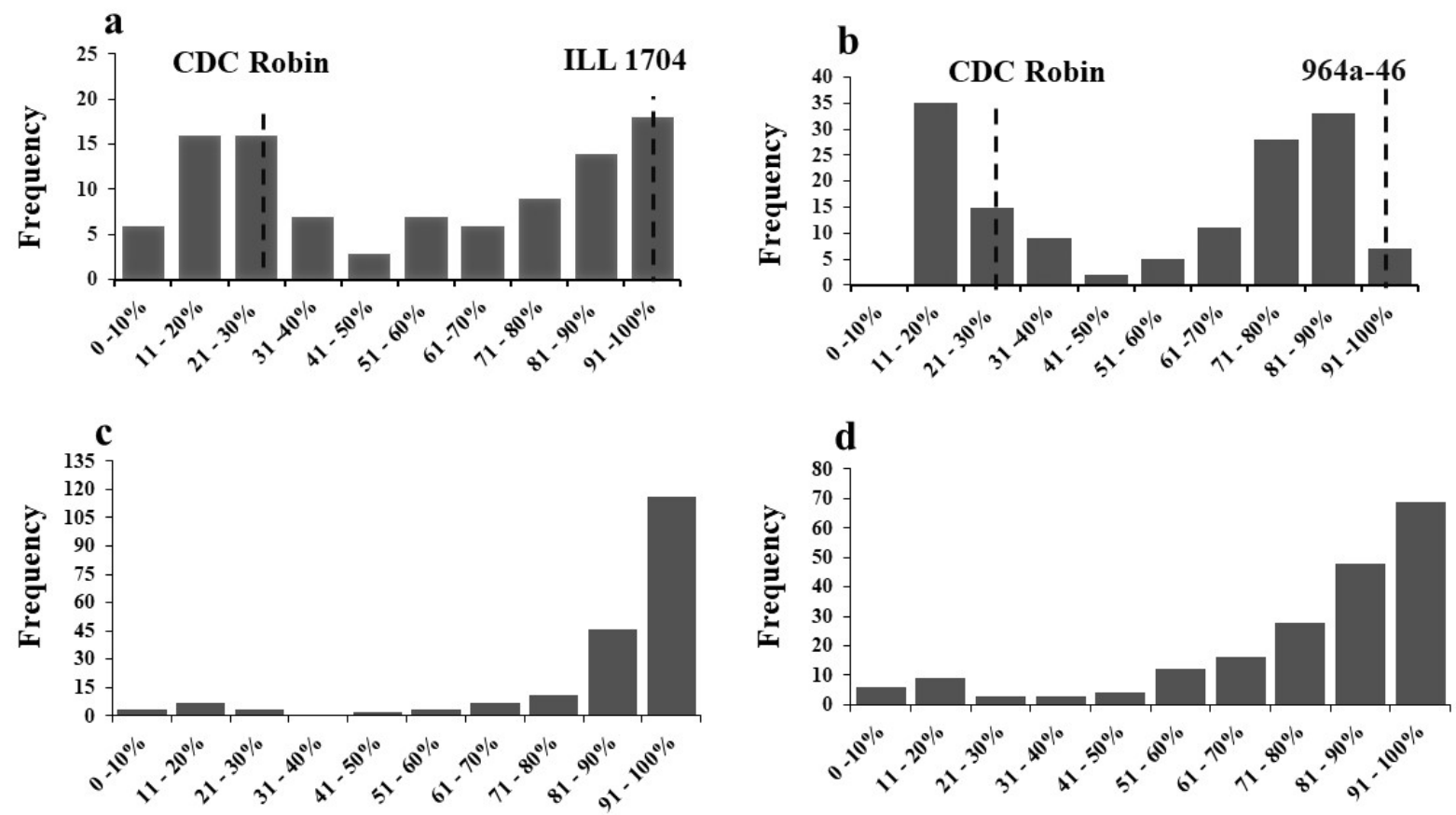

Disease severity score

\section{Disease severity score}


Figure 1. Frequency distribution of anthracnose race 1 severity of; (a) 102 RILs of LR-01 (ILL $1740 \times$ CDC Robin), and (b) 139 RILs of LR-18 (CDC Robin $\times$ 964a-46) in growth chamber conditions. (c, d) frequency distributions of the 200 lentil genotypes in the GWAS panel evaluated under growth chamber and polyhouse conditions. The vertical lines indicate the average disease severity of the parents. Disease severity was rated on a 0-10 scale, where the disease severity score increased in $10 \%$ increments.

The disease severity distribution for the GWAS panel was skewed towards susceptibility in both testing environments (Figure $1 \mathrm{c}$ and $\mathrm{d}$ ). Under growth chamber conditions, 6.5, 8.5, and 85\%; and for polyhouse conditions, 9, 25.5 and $65.5 \%$ of the genotypes had resistant, intermediate, and susceptible reactions, respectively. The results suggest the presence of limited sources of resistance among most of the genotypes tested against race 1 , even though it was less aggressive than $C$. lentis race 0 (Buchwaldt et al., 2004). The differences in disease severity scores among the genotypes in each environments and genotype $\times$ environment interactions were significant (Table 1). The estimated broad-sense heritability was high, 0.96 for growth chamber, 0.88 for polyhouse and 0.92 for combined analysis of both environments (Table 1), demonstrating that race 1 resistance was controlled by genetic factors and that the data could be used for accurate mapping of race 1 resistance genes. A positive correlation (Spearman $r=0.61, p<0.001$ ) was observed between growth chamber and polyhouse data for race 1 response of genotypes. 
Table 1. Analysis of variance components for anthracnose race 1 severity of 200 lentil genotypes evaluated under growth chamber and polyhouse conditions.

\begin{tabular}{lllllll}
\hline Environment & Mean & Range & $\mathbf{h}_{\mathbf{B}}{ }^{\mathbf{2}}$ & $\boldsymbol{\sigma}^{\mathbf{2}} \mathbf{G}$ & $\boldsymbol{\sigma}^{\mathbf{2}} \mathbf{G E}$ & $\boldsymbol{\sigma}_{\mathbf{e}}$ \\
\hline Growth chamber & 84.2 & $6.4-95$ & 0.96 & $405.1^{* * *}$ & - & 104.8 \\
Polyhouse & 76.0 & $5.0-95$ & 0.88 & $454.5^{* * *}$ & - & 180.5 \\
Combined & 80.1 & $5.7-95$ & 0.92 & $382.8^{* * *}$ & $45.55^{* * *}$ & 133.2
\end{tabular}

*** $p<0.0001, \sigma_{\mathrm{G}}^{2}$, genotypic variance; $\sigma_{\mathrm{GE}}^{2}$, genotype $\times$ environment variance; $\sigma^{2} \mathrm{e}$, error variance; $\mathrm{h}_{\mathrm{B}}^{2}$, heritability.

\section{Linkage map construction for the LR-01 population}

Seven linkage groups (LG) were constructed from the segregation patterns of 21,634 SNPs. LGs were assigned to their respective chromosomes based on where markers lie in the reference genome. The detailed information for the LR-01 linkage map is provided in supplemental Table S2. The number of SNPs assigned to each linkage group (LG) ranged from 1710 SNP markers (LG 7) to 5120 (LG 2). After binning of SNPs with redundant information, the 21,634 SNPs were grouped into 921 recombination bins and 1807 single markers. In total, 2728 informative loci distributed along the seven LGs were retained on the LR-01 linkage map. The map covered a total length of $1643.8 \mathrm{cM}$, with an average distance between the neighboring SNP markers of $0.6 \mathrm{cM}$. The length of each LG varied from $164.6 \mathrm{cM}$ for LG 7 to $299.5 \mathrm{cM}$ for LG 3.

\section{QTL mapping of anthracnose race 1 resistance}

A single significant QTL conferring resistance to anthracnose race 1 was identified on chromosome 3 using both LR-01 and LR-18 RIL populations and designated as qAnt1.Lc-3 (Figure 2). On the LR-18 linkage map, the QTL was flanked by SNP markers LcC03673p249/LcC03441p105 and LcC09426p518, with an interval ranging from 43.2 to 51.5 
cM. The SNP markers were mapped within a $7.6 \mathrm{Mb}$ (30704841 to $38275723 \mathrm{bp})$ physical interval on the CDC Redberry genome assembly v.2.0 (Ramsay et al., 2019). For the LR-01 population genetic map, qAnt1.Lc-3 mapped to an interval of $53.2 \mathrm{cM}$ to $70.8 \mathrm{cM}$ corresponding to a physical location of 29313444 to 38758654 bp (9.4 Mb region) on chromosome 3. Importantly, the highdensity genetic map of the LR-01 population contains a number of SNP markers associated with race 1 resistance in the interval of the QTL region. Among these, a cluster of markers mapped to Lcu.2RBY.unitig0289 which is unanchored in the reference genome, suggesting it should be part of chromosome 3. The percentage of variation in race 1 resistance explained by $q$ Ant $1 . L c-3$ varied from 66.6 to $69.8 \%$, with a LOD score value ranging from 24.3 to 37.1 (Table 2). As expected, CDC Robin (the resistant parent) contributed the resistance allele for qAnt1.Lc-3.

Table 2. QTL mapping of anthracnose race 1 resistance detected by multiple QTL models of R/qtl in two biparental RIL populations: LR-01 (ILL $1704 \times$ CDC Robin) and LR-18 (CDC Robin $\times$ $964 a-46)$.

\begin{tabular}{|c|c|c|c|c|c|c|c|c|}
\hline Population & $\mathrm{QTL}^{\epsilon}$ & $\mathrm{LG}^{\#}$ & $\begin{array}{l}\text { Peak } \\
\text { LOD }\end{array}$ & $\begin{array}{l}\text { Position } \\
\text { (cM) }\end{array}$ & $\begin{array}{c}1.5 \mathrm{LOD} \\
\text { interval }(\mathrm{cM})\end{array}$ & $\begin{array}{c}\text { Position }^{@} \\
\text { (Mb) }\end{array}$ & $\begin{array}{l}\mathrm{PVE}^{¥} \\
(\%)\end{array}$ & $\mathrm{Add}^{\$}$ \\
\hline LR-01 & & 3 & 24.3 & 60.9 & $53.2-70.8$ & $29.3-38.8$ & 66.6 & -25.9 \\
\hline LR-18 & $q A n t 1 . L c-3$ & 3 & 35.1 & 44.1 & $43.2-51.5$ & $30.7-38.8$ & 69.8 & -26.6 \\
\hline
\end{tabular}


LR-18 - LG 3

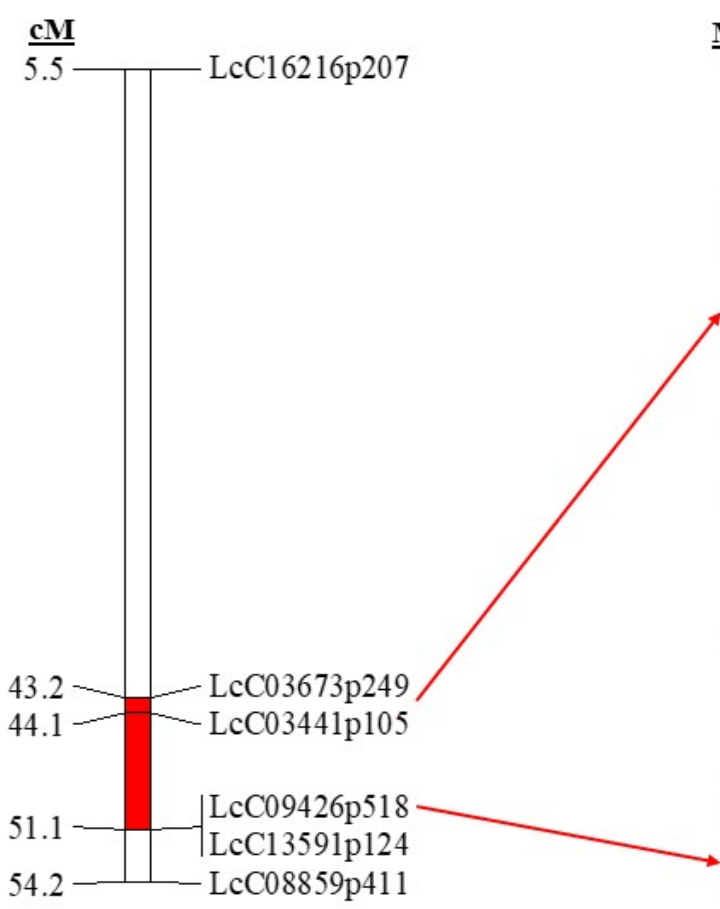

Chromosome 3

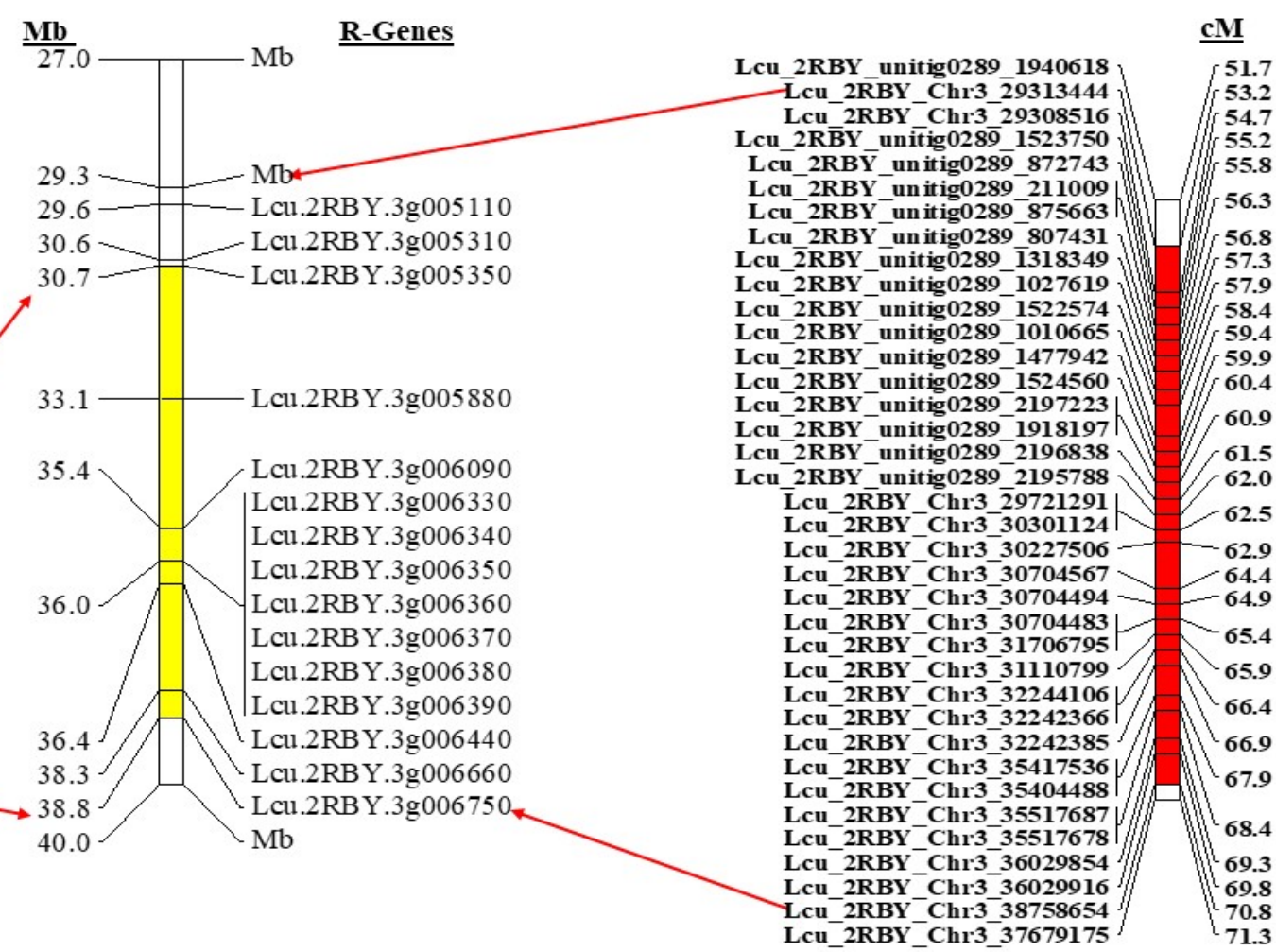

Figure 2. Position of anthracnose race 1 resistance QTL on linkage group (LG) 3 of the recombinant inbred line (RIL) populations LR18 (CDC Robin $\times$ 964a-46; left) and LR-01 (ILL $1704 \times$ CDC Robin; right) evaluated under growth chamber conditions. The red regions on the bar highlights the QTL interval on LGs; and the yellow region depicts the interval overlapped for both LGs on lentil chromosome 3 (Ref. genome v2.0) and the predicted candidate disease resistance genes (R-genes) is on the right. The positions are in centimorgan $(\mathrm{cM})$ and mega base pairs $(\mathrm{Mb})$ as indicated on the top of the bar 


\section{Genome-wide association study of anthracnose race 1 resistance}

A total of 152,011 SNP markers were used for marker-trait association analysis. The number of SNP markers per chromosome varied from 16,848 on chromosome 7 to 26,349 on chromosome 2 (Supplemental Figure S1). The average physical distance between two markers used in this study was approximately $26 \mathrm{~kb}$, across a genome size of $\sim 4 \mathrm{~Gb}$ with a mean of $21,716 \mathrm{SNP}$ markers per chromosome. The SNP markers were evenly distributed and adequately covered the genome for the purpose of GWAS analysis.

The model-based population structure analysis revealed that the 200 lentil genotypes could be grouped into three major subpopulations, and that finer hierarchical structures were evident in the diversity panel (Supplemental Figure S2 a). Using $\mathrm{k}=3,90.5 \%$ of the accessions were assigned to three groups, and only $9.5 \%$ of the accessions were assigned to mixed populations (Supplemental Figure S2 b). The principal component analysis (PCA) showed that the variance explained by the eigenvalue of each principal component (PC) dropped rapidly after the first three PCs, which explained approximately $35 \%$ of the total genetic variances for the diversity panel (Supplemental Figure S2 c). The results of the PCA were consistent following STRUCTURE analyses (Supplemental Figure S2 d). Thus, the first three PCAs were used as a covariate in the mixed linear model in the GWAS analysis and were also confirmed by visual inspection of Q-Q plots (Supplemental Figure S3).

GWAS analysis using the combined disease severity data detected 14 SNPs that were significantly associated with race 1 resistance in the lentil genome $\left(-\log _{10}(\mathrm{p}) \geq 5.2\right)$ (Figure 3; Table 3). The GWAS analysis for individual environments identified 26 and 11 SNPs associated with race 1 resistance under growth chamber and polyhouse conditions, respectively (Figure 3 and 
Supplemental Table S3). Most of the loci detected were common between both environments with varying $p$-values of SNPs associated with the loci. The phenotypic variation $\left(\mathrm{R}^{2}\right)$ explained by an individual significant SNP marker ranged from 58 to $69 \%$.
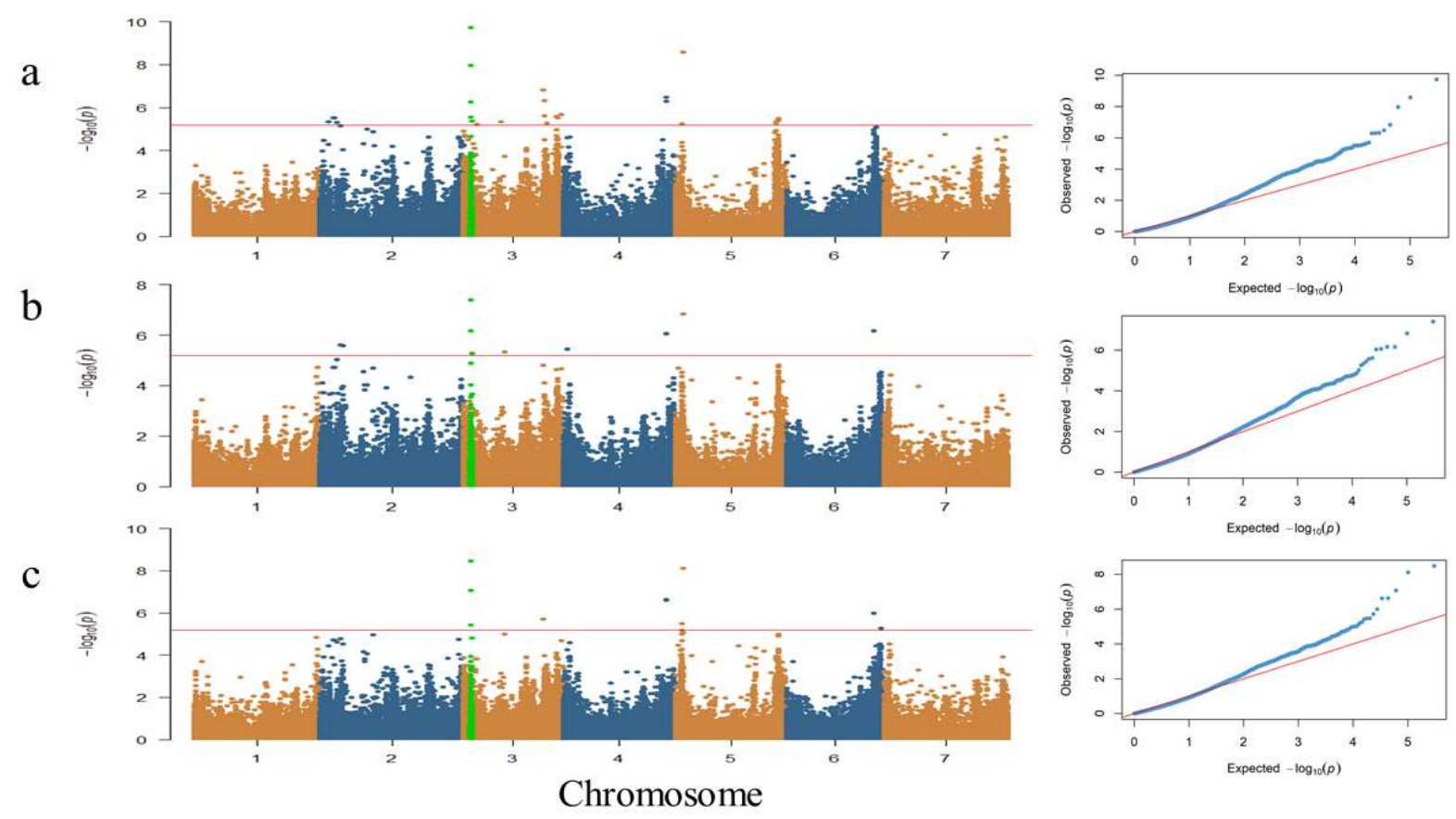

Figure 3. Manhattan and Quantile-quantile (Q-Q) plots of genome wide association study (GWAS) for anthracnose race 1 resistance in 200 lentil accessions evaluated in (a) the growth chamber, (b) the polyhouse and (c) the combined lsmean of disease severity scores from both environments. Alternating colors indicate the 7 chromosomes and the $Y$-axis indicates $-\log _{10}$ of $\mathrm{p}$ values with significant association at 5.2 (red line). The green dots on chromosome 3 represent the SNP marker in the QTL (qAnt1.Lc-3) interval from biparental populations.

The SNPs previously associated with the $q A n t 1 . L c-3$ region on chromosome 3 were again the most significant with $-\log _{10}(p)=9.6$ and $\mathrm{R}^{2}=69 \%$ (Figure 3 and Table 3 ) and were detected in all analyses. This region was tagged with four significant SNP markers (Lcu.2RBY.Chr3.33827173, Lcu.2RBY.Chr3.33827185, Lcu.2RBY.Chr3.34117023 and Lcu.2RBY.Chr3.35384298) and 
spanned 1.6 Mb. Two other genomic regions on chromosome 3 displayed a peak SNP marker at 341.3 $\mathrm{Mb}\left(\mathrm{R}^{2}=66 \%\right)$ and 417.9 $\mathrm{Mb}\left(\mathrm{R}^{2}=65 \%\right)$ for combined and growth chamber analysis. Two SNP markers associated with race 1 resistance were detected on chromosome 4 within 4 bp (442702129 bp to $442702133 \mathrm{bp}$ ) in all analyses. Two other significant regions were identified for combined and growth chamber data on chromosome 5 from 28.4 to $33.7 \mathrm{Mb}$ for all analysis and from 427.5 to $437.9 \mathrm{Mb}$. One SNP marker located at 374.3 Mb on chromosome 6 was detected for the combined and the polyhouse analyses (Table 3).

Table 3. SNP markers associated with anthracnose race 1 resistance using combined lsmean data of disease severity from growth chamber and polyhouse for a set of 200 lentil accessions.

\begin{tabular}{lccccc}
\hline \multicolumn{1}{c}{ SNP Marker } & Chr & ${\text { Position }(\mathrm{Mb})^{\#}}^{*}$ & $P$. value & MAF & $\mathrm{R}^{2 \$}$ \\
\hline Lcu.2RBY.Chr3.33827173 & 3 & 33827173 & $1.38 \mathrm{E}-06$ & 0.16 & 0.65 \\
Lcu.2RBY.Chr3.33827185 & 3 & 33827185 & $2.43 \mathrm{E}-08$ & 0.15 & 0.67 \\
Lcu.2RBY.Chr3.34117023 & 3 & 34117023 & $2.47 \mathrm{E}-10$ & 0.14 & 0.69 \\
Lcu.2RBY.Chr3.35384298 & 3 & 35384298 & $3.24 \mathrm{E}-06$ & 0.14 & 0.65 \\
Lcu.2RBY.Chr3.341261994 & 3 & 341261994 & $4.30 \mathrm{E}-07$ & 0.23 & 0.66 \\
Lcu.2RBY.Chr3.417940994 & 3 & 417940994 & $6.13 \mathrm{E}-06$ & 0.06 & 0.64 \\
Lcu.2RBY.Chr4.442702129 & 4 & 442702129 & $8.32 \mathrm{E}-08$ & 0.11 & 0.66 \\
Lcu.2RBY.Chr4.442702133 & 4 & 442702133 & $8.48 \mathrm{E}-08$ & 0.11 & 0.66 \\
Lcu.2RBY.Chr5.28582530 & 5 & 28582530 & $3.74 \mathrm{E}-06$ & 0.12 & 0.65 \\
Lcu.2RBY.Chr5.28637458 & 5 & 28637458 & $3.74 \mathrm{E}-06$ & 0.12 & 0.65 \\
Lcu.2RBY.Chr5.33721990 & 5 & 33721990 & $1.82 \mathrm{E}-09$ & 0.21 & 0.68 \\
Lcu.2RBY.Chr5.437910070 & 5 & 437910070 & $3.23 \mathrm{E}-06$ & 0.13 & 0.65 \\
Lcu.2RBY.Chr5.437944230 & 5 & 437944230 & $3.95 \mathrm{E}-06$ & 0.13 & 0.65 \\
Lcu.2RBY.Chr6.374326758 & 6 & 374326758 & $8.88 \mathrm{E}-07$ & 0.11 & 0.65 \\
\hline
\end{tabular}

${ }^{\#}$ Physical positions, ${ }^{\$}$ Explained phenotypic variance per marker 


\section{Candidate gene prediction}

We explored the candidate genes associated with anthracnose race 1 resistance in the region of qAnt1.Lc-3 physical genomic intervals that overlapped for both genetic maps and the GWAS. The interval covered $8 \mathrm{Mb}$ from 30.0 - 38.0 Mb on chromosome 3 and contains 119 annotated genes. Among these, 11 genes encode for typical resistance (R) genes, which include nucleotide-bindingleucine-rich repeat (NB-LRR) domain disease resistance proteins (Table 4), and 46 are known to be involved in defense response reactions to pathogens and other stresses (Supplemental Table S4). Moreover, two NB-LRR domain genes (Lcu.2RBY.L001220 and Lcu.2RBY.L001240) were tagged by SNPs in unitig Lcu.2RBY.unitig0289 but mapped in the QTL region of the LR-01 genetic map, providing further evidence that they possibly correspond to a chromosome 3 NBLRR domain cluster. Within the qAnt1.Lc-3 region, the most significant GWAS SNPs include one located $103 \mathrm{~kb}$ upstream of the gene encoding an anthranilate N-benzoyltransferase protein (Lcu.2RBY.3g005860), two significant SNPs within genes involved with cellulose synthase (Lcu.2RBY.3g005880), and one significant SNP located within a gene encoding for a disease resistance protein TIR-NBS-LRR domain (Lcu.2RBY.3g006090). Almost all the significant GWAS SNPs identified are located within or close to an annotated gene (Supplemental Table S4). 
Table 4. A subset of candidate resistance genes associated with anthracnose race 1 resistance identified in the interval of QTL and GWAS regions according to gene annotation.

\begin{tabular}{cllll}
\hline Chr\$ & Gene ID & \multicolumn{2}{l}{ Physical position (bp) } & Annotation \\
\hline Chr3 & Lcu.2RBY.3g005310 & 30641401 & 30646173 & NB-ARC domain disease resistance protein \\
Chr3 & Lcu.2RBY.3g005350 & 30735946 & 30736590 & Wall-associated receptor kinase-like protein \\
Chr3 & Lcu.2RBY.3g005880 & 33819256 & 33828131 & Cellulose synthase \\
Chr3 & Lcu.2RBY.3g005910 & 34117126 & 34118497 & Anthranilate N-benzoyltransferase \\
Chr3 & Lcu.2RBY.3g006090 & 35383081 & 35387584 & TIR-NBS-LRR class disease resistance protein \\
Chr3 & Lcu.2RBY.3g006330 & 35972988 & 35973704 & LRR \& NB-ARC domain disease resistance protein \\
Chr3 & Lcu.2RBY.3g006340 & 35974000 & 35976197 & LRR \& NB-ARC domain disease resistance protein \\
Chr3 & Lcu.2RBY.3g006350 & 35976249 & 35981698 & LRR \& NB-ARC domain disease resistance protein \\
Chr3 & Lcu.2RBY.3g006360 & 35981709 & 35982218 & NB-ARC domain disease resistance protein \\
Chr3 & Lcu.2RBY.3g006370 & 35982494 & 35982865 & CC-NBS-LRR resistance protein \\
Chr3 & Lcu.2RBY.3g006380 & 36028314 & 36032907 & LRR \& NB-ARC domain disease resistance protein \\
Chr3 & Lcu.2RBY.3g006390 & 36037105 & 36044777 & LRR \& NB-ARC domain disease resistance protein \\
Unitig0289 & Lcu.2RBY.L001220 & 1917198 & 1921007 & LRR \& NB-ARC domain disease resistance protein \\
Unitig0289 & Lcu.2RBY.L001240 & 1939874 & 1942161 & NB-ARC domain disease resistance protein
\end{tabular}

${ }^{\#}$ Position in bp according to CDC Redberry genome ref. v.2.0, ${ }^{\beta}$ Genes identified with GWAS SNPs, ${ }^{\$}$ Chromosome 


\section{DISCUSSION}

Developing host resistance is the most preferable, economical, and sustainable strategy for managing anthracnose in lentil production. However, the process requires sufficient information about genetic sources of resistance and identification of resistance loci associated with races of anthracnose to use marker assisted breeding strategy for gene pyramiding. We used a combination of traditional QTL mapping and GWAS to increase our understanding of anthracnose race 1 resistance in lentil. To our knowledge, this study is the first on anthracnose race 1 resistance in lentil to employ biparental QTL mapping and GWAS using a large number of physical SNP marker positions.

Analysis of the differential responses of the RIL populations and GWAS accessions to race 1 inoculation provided frequency distributions and heritability estimates of the inheritance of disease resistance. The disease reactions of the RILs of both populations displayed a bimodal distribution that fit a 1:1 ratio indicating a Mendelian one gene model, indicative of a major QTL identified in the populations. Analysis of variance for the GWAS panel revealed significant variation among genotypes. However, disease reaction frequencies of the lines showed a skewed distribution to greater susceptibility. The narrow genetic base for anthracnose resistance is evident in current collections of lentil accessions from different gene banks. For example, previous evaluations of resistance to anthracnose race 1 of lentil accessions from 50 countries identified $16(0.9 \%)$ of 1771 (Buchwaldt et al., 2004), and 15 (2.6\%) of 579 (Shaik et al., 2013). This may be attributable to genetic bottlenecks that were created at the time of domestication (Sonnante et al., 2009) due to the absence of the disease in its region of domestication. Until recently, the disease was considered minor or had not been reported in other parts of the world (Banniza et al., 2018), including its 
center of origin and/or diversity where lentil has been grown for centuries. Conversely, the rapid expansion of the lentil crop in the prairie ecosystem, particularly in the Western Canadian production system, coincides with the incidence of anthracnose (Morrell, 1997). Tanksley and McCouch (1997) argued expansion of a few improved cultivars into a modern agricultural system can result in emergence of new disease threats that can occur due to plant pathogens host shift (Silva et al., 2012).

Breeding for partial resistance to anthracnose race 1 in lentil started with release of the cultivar CDC Robin (Vandenberg et al., 2002), which was derived from PI 320952 (Indianhead). The resistance in PI 320952 was shown to be governed by a recessive and a closely linked dominant gene (Buchwaldt et al., 2013). In this study, we mapped a major QTL (qAnt1.Lc-3) associated with race 1 resistance from CDC Robin on chromosome 3 in both populations. The QTL qAnt1.Lc-3 accounts for $66.6-69.8 \%$ of the variance in resistance to race 1. Bhadauria et al. (2017) reported race 1 resistance QTL on chromosomes 2, 3, and 5 of the L. ervoides genome. We do not have sufficient information to determine if the QTL on chromosome 3 of L. ervoides is the same as that in the cultivated lentil but both genomes are largely collinear along their length of this chromosome (Ramsay et al., unpublished data). Large portions of the physical interval of qAnt1.Lc-3 were colocalized for the LR-01 and LR-18 population genetic maps, indicating a strong association of the genomic region with race 1 resistance.

The association mapping approach is suited for the detection of high-resolution QTLs, as it captures a larger portion of the recombination events that have accumulated inside an association panel (Zhu et al., 2008). Exploration of high throughput marker data makes GWAS more efficient 
and provides a rapid method to identify significant genomic regions associated with traits of interest for candidate gene prediction (Yano et al., 2016). In the current study, marker-trait associations identified 14 SNPs associated with race 1 resistance on chromosomes 3, 4, 5 and 6 . Most of these MTA were consistent across test conditions, explained by the high heritability estimates and the high correlation observed between results from growth chamber and polyhouse environments. Chongo and Bernier (1999) also reported significant correlation between field inoculations and anthracnose screening under controlled conditions for resistant L. culinaris germplasm. The major locus on chromosome 3 identified using the biparental populations was confirmed by GWAS. The most significant SNPs associated with race 1 resistance were located within the region of $q A n t 1 . L c-3$. The GWAS SNPs fine mapped the $q A n t 1 . L c-3$ region to $1.6 \mathrm{Mb}$ containing candidate resistance genes.

A total of 57 candidate genes involved in plant defense against biotic and abiotic stress are predicted within the region of $q A n t 1 . L c-3$ identified in the biparental mapping (Supplemental Table S4). Among these, 13 are NB-LRR class R genes, and 14 are transmembrane protein (TM) genes known as 'oth-R' genes (Sanseverino and Ercolano, 2012; Sekhwal et al., 2015). In plant genomes, the NB-LRR class R genes are abundant and often clustered on specific chromosomes due to tandem and segmental duplications (Leister, 2004). Congruently, most of the annotated NBS-LRR genes in the lentil genome are located on chromosome 3 (Ramsay et al., unpublished data). Thus, a cluster of candidate $\mathrm{R}$ genes identified within the region of qAnt $1 . L c-3$ could possibly account for improved resistance to race 1 in lentil. Transmembrane (TM) proteins are part of a plant cell complex membrane-associated receptors that mediate signal transduction between the extra- and intracellular environments in defense system and are mainly involved in conferring 
a broader resistance spectrum in plants, including the known Mlo gene (Büschges et al., 1997;

Xiao et al., 2001; Brandwagt et al., 2002; Ma et al.,2014). The most significant SNP markers from

GWAS analysis also identified genes involved with cellulose synthase and anthranilate Nbenzoyltransferase protein within $q A n t 1 . L c-3$. Cellulose synthases play an important role in mediating cell wall changes in the epidermal layers in response to defense against pathogens (Douchkov et al., 2016). Anthranilate N-benzoyltransferase genes are important in phytoalexins biosynthesis, which provide enhanced protections against pathogens (Grayer and Kokubun, 2001).

In conclusion, we combined the use of biparental QTL mapping and GWAS to identify QTL and candidate genes for anthracnose race 1 resistance in lentil. The major effect QTL (qAnt1.Lc-3) identified on chromosome 3, explained 66.6 to $69.8 \%$ of the phenotypic variance and was confirmed via GWAS analysis. Across the genome, GWAS identified 14 SNPs associated with race 1 resistance. The SNP markers identified that were associated with the candidate genes can be used for MAS to advance molecular breeding approaches for improving anthracnose resistance in lentil.

\section{SUPPLEMENTAL MATERIAL}

Four supplemental tables (Supplemental Table S1 - S4) and three supplemental figures (Supplemental Figure S1-S3) are included as supporting information.

\section{ACKNOWLEDGEMENTS}

The authors gratefully acknowledge funding from the Natural Sciences and Engineering Research Council of Canada (NSERC) Industrial Research Chair Program, the Saskatchewan Pulse Growers, and the University of Saskatchewan. We are grateful for financial support from a project 'Application of Genomics to Innovation in the Lentil Economy (AGILE)' funded by Genome Canada, Saskatchewan Pulse Growers, Western Grains Research Foundation, and the Province of 
Saskatchewan, and managed by Genome Prairie. We are also thankful for the technical assistance of the bioinformatics, molecular and pathology lab staff of the Pulse Breeding and Genetics group at the University of Saskatchewan.

\section{CONFLICTS OF INTEREST}

The authors declare that they have no competing interests.

\section{AUTHOR CONTRIBUTIONS}

TSG generated phenotypic data, performed data analyses, and wrote the manuscript. LR conducted SNP calling, TAH constructed the genetic map (LR-01), AV and KEB participated in experimental design and reviewed the manuscript. All authors read and approved the submitted version.

\section{DATA AVAILABILITY}

The raw genotypic data supporting this study are available at https://knowpulse.usask.ca/Geneticmap/2686922; https://knowpulse.usask.ca/Geneticmap/2695342 and https:// knowpulse.usask.ca/Lentil-AGILE-project or from the authors upon request. The SNPs that showed significant associations with anthracnose race 1 resistance are provided in Supplemental Information.

\section{REFERENCES}

Aldemir, S., Ateş, D., Temel, H. Y., Yağmur, B., Alsaleh, A., Kahriman, A., Özkan, H., Vandenberg, A., \& Tanyolac, M.B. (2017). QTLs for iron concentration in seeds of the cultivated lentil (Lens culinaris Medik.) via genotyping by sequencing. Turkish Journal of Agriculture and Forestry, 41, 243-255.

Ali, S. (1995). Register of Australian grain legume cultivars. Lens culinaris (lentil) cv. Northfield. Australian Journal of Experimental Agriculture, 35, 1181-1182.

Arumuganathan, K., \& Earle, E.D. (1991). Nuclear DNA content of some important plant species. Plant Molecular Biology Reporter, 9, 208-218.

Ates, D., Sever, T., Aldemir, S., Yagmur, B., Temel, H. Y., Kaya, H. B., ... Tanyolac, B. (2016). Identification QTLs controlling genes for Se uptake in lentil seeds. PLoS ONE, 11, e0149210. 
Banniza, S., Warale, R., Meant, J., Cohen-Skali, A., Armstrong-Cho, C., \& Bhadauria, V. (2018). The long path to understanding the host-pathogen interactions of Colletotrichum lentis on lentil. Canadian Journal of Plant Pathology, 40(2), 199-209.

Bates, D., Mächler, M., Bolker, B., \& Walker, S. (2015). Fitting Linear Mixed-Effects Models Using lme4. Journal of Statistical Software, 67, 1-48.

Bayaa, B., \& Erskine, W. (1997). Diseases of lentil. In: Alleen, D. J., Lenné J. M. (eds). The pathology of food and pasture legumes. Wallingford: CAB international in association with Patancheru: ICRISAT; p. 423-471.

Bellar, M., and Kebabeh, S. (1983). A list of diseases, injuries, and parasitic weeds of lentils in Syria (survey 1979-80). LENS Newsletter 10:30-31.

Bhadauria, V., Ramsay, L., Bett, K. E., \& Banniza, S. (2017). QTL mapping reveals genetic determinants of fungal disease resistance in the wild lentil species Lens ervoides. Scientific Reports, 7, 3231.

Brandwagt, B. F., Kneppers, T. J., Nijkamp, H. J., \& Hille, J. (2002). Overexpression of the tomato Asc-1 gene mediates high insensitivity to AAL toxins and fumonisin B1 in tomato hairy roots and confers resistance to Alternaria alternata f. sp. lycopersici in Nicotiana umbratica plants. Molecular Plant-Microbe Interactions, 15, 35-42.

Broman, K. W., Wu, H., Sen, S., \& Churchill, G. A. (2003). R/qtl: QTL mapping in experimental crosses. Bioinformatics, 19, 889-890.

Buchwaldt, L., Anderson, K. L., Morrall, R. A. A., Gossen, B. D., \& Bernier, C. C. (2004). Identification of lentil germplasm resistant to Colletotrichum truncatum and characterization of two pathogen races. Phytopathology, 94, 236-243.

Buchwaldt, L., Dzananovic, E., \& Durkin, J. (2018). Lentil anthracnose: epidemiology, fungicide decision support system, resistance and pathogen races. Canadian Journal of Plant Pathology, 40(2), 189-198.

Buchwaldt, L., Shaikh, R., Adam, J., Tullu, A., \& Slinkard, A. E. (2013). Recessive and dominant genes confer resistance to Colletotrichum truncatum in cultivated lentil. Canadian Journal of Plant Pathology, 35, 222-231.

Büschges, R., Hollricher, K., Panstruga, R., Simons, G., Wolter, M., Frijters, A., ... SchulzeLefert, P. (1997). The barley Mlo gene: A novel control element of plant pathogen resistance. Cell, 88, 695-705.

Chongo, G., \& Bernier, C. C. (1999). Field and growth chamber evaluation of components of partial resistance to Colletotrichum truncatum in lentil. Canadian Journal of Plant Pathology, 21:58-63.

Chongo, G., Bernier, C. C., \& Buchwaldt, L. (1999). Control of anthracnose in lentil using partial resistance and fungicide applications. Canadian Journal of Plant Pathology, 21, 16-22.

Damm, U., O’Connell, R. J., Groenewald, J. Z., \& Crous, P.W. (2014), The Colletotrichum destructivum species complex-hemibiotrophic pathogens of forage and field crops. Studies in Mycology, 79, 49-84. 
Danecek, P., Auton, A., Abecasis, G., Albers, C. A., Banks, E., DePristo, M. A., ... Durbin, R. (2011). The Variant Call Format and VCFtools. Bioinformatics, 27, 2156-2158.

Douchkov, D., Lueck, S., Hensel, G., Kumlehn, J., Rajaraman, J., Johrde, A, ... Schweizer, P. (2016). The barley (Hordeum vulgare) cellulose synthase-like D2 gene (HvCsiD2) mediates penetration resistance to host-adapted and nonhost isolates of the powdery mildew fungus. New Phytologist, 212, 421-433.

Earl, D., \& VonHoldt, B. (2012). STRUCTURE HARVESTER: a website and program for visualizing STRUCTURE output and implementing the Evanno method. Conservation Genetics Resources, 4, 359-361.

Falush, D., Stephens, M., \& Pritchard, J. K. (2003). Inference of population structure using multilocus genotype data: linked loci and correlated allele frequencies. Genetics, 164: $1567-1587$.

Food and Agriculture Organization of the United Nations (2021). FAOSTAT, Crops. http://faostat3.fao.org (Accessed: 9th January 2021).

Fedoruk, M. J., Vandenberg, A., \& Bett, K. E. (2013). Quantitative trait loci analysis of seed quality characteristics in lentil using single nucleotide polymorphism markers. Plant Genome, 6, 1-10.

Gela, T. S., Banniza, S., \& Vandenberg, A. (2020). Lack of effective resistance to the virulent race of Colletotrichum lentis in Lens culinaris Medikus subsp. culinaris. Plant Genetic Resources: Characterization and Utilization, 18, 81-87.

Government of Saskatchewan. Varieties of grain crops. (2019). http://publications.gov.sk.ca/details.cfm?p=83696.

Grayer, R. J., \& Kokubun, T. (2001). Plant-fungal interactions: the search for phytoalexins and other antifungal compounds from higher plants. Phytochemistry, 56, 253-261.

Haile, T. A., Heidecker, T., Wright, D., Neupane, S., Ramsay, L., Vandenberg, A., \& Bett, K. E. (2020). Genomic selection for lentil breeding: Empirical evidence. Plant Genome, e20002.

Haley, C. S., \& Knott, S. A. (1992). A simple regression method for mapping quantitative trait loci in line crosses using flanking markers. Heredity, 69, 315-324.

Kaiser, W.J., Mihov, M., Muehlbauer, F.J., \& Hannan, R.M. (1998). First report of anthracnose of lentil incited by Colletotrichum truncatum in Bulgaria. Plant Disease, 82,128.

Khazaei, H., Fedoruk, M., Caron, C. T., Vandenberg, A., \& Bett, K. B. (2018). Single nucleotide polymorphism markers associated with seed size quality characteristics of cultivated lentil. Plant Genome, 11, 1-7.

Kissinger, G. (2016). Pulse crops and sustainability: A framework to evaluate multiple benefits. http://www.fao.org/pulses.

Knapp, S. J., Stroup, W. W., and Ross, W. M. (1985). Exact confidence intervals for heritability on a progeny mean basis. Crop Science, 25, 192-194.

Kosambi, D. D. (1944). The estimation of map distances from recombination values. Annals of Eugenics, 12, 172-175. 
Kumar, H., Singh, A., Dikshit, H. K., Mishra, G. P., Aski, M., Meena, M. C., \& Kumar, S. (2019). Genetic dissection of grain iron and zinc concentrations in lentil (Lens culinaris Medik.). Journal of Genetics, 98, 66.

Kumar, J., Gupta, S., Gupta, D. S., \& Singh, N.P. (2018). Identification of QTLs for agronomic traits using association mapping in lentil. Euphytica, 214, 75.

Kumar, S., Rajendran, K., Kumar, J., Hamwieh, A., \& Baum, M. (2015). Current knowledge in lentil genomics and its application for crop improvement. Frontiers in Plant Science, 6, 78.

Leister, D. (2004). Tandem and segmental gene duplication and recombination in the evolution of plant disease resistance genes. Trends in Genetics, 20, 116-122.

Li, G., Xu, X., Tan, C., Carver, B., Bai, G., Wang, X., ... Cowg, C. (2019). Identification of powdery mildew resistance loci in wheat by integrating genome-wide association study (GWAS) and linkage mapping. Crop Journal, 7, 294-306.

Lipka, A. E., Tian, F., Wang, Q., Peiffer, J., Li, M., Bradbury, P. J, ... Zhang, Z. (2012). GAPIT: genome association and prediction integrated tool. Bioinformatics, 28, 2397-2399.

Ma, X. F., Li, Y., Sun, J. L., Wang, T. T., Fan, J., Lei, Y., ...Wang, W. M. (2014). Ectopic expression of resistance to powdery mildew8.1 confers resistance to fungal and oomycete pathogens in Arabidopsis. Plant Cell Physiology, 55, 1484-1496.

Manichaikul, A., Moon, J. Y., Sen, S., Yandell, B. S., \& Broman, K. W. (2009). A model selection approach for the identification of quantitative trait loci in experimental crosses, allowing epistasis. Genetics, 181, 1077-1086.

Meng, L., Li, H., Zhang, L., \& Wang, J. (2015). QTL IciMapping: integrated software for genetic linkage map construction and quantitative trait locus mapping in biparental populations. Crop Journal, 3, 269-283.

Morrall, R. A. A., \& Pedersen, E. A. (1991). Discovery of lentil anthracnose in Saskatchewan in 1990. Canadian Journal of Plant Pathology, 71, 105-106.

Morrall, R. A.A. (1997). Evolution of lentil diseases over 25 years in western Canada. Canadian Journal of Plant Pathology, 19, 197-207.

Ogutcen, E., Ramsay, L., von Wettberg, E. B., \& Bett, K. E. (2018). Capturing variation in Lens (Fabaceae): Development and utility of an exome capture array for lentil. Applications in Plant Sciences, 6, e01165.

Patterson, N., Price, A. L, \& Reich, D. (2006). Population structure and eigen analysis. PLoS Genetics, 2, e190.

Polanco, C., Sa'enz de Miera, L. E., Gonza'lez, A. I., Garcı'a, P., Fratini, R., Vaquero, F., ... De la Vega, M. P. (2019). Construction of a high-density interspecific (Lens culinaris $\times L$. odemensis) genetic map based on functional markers for mapping morphological and agronomical traits, and QTLs affecting resistance to Ascochyta in lentil. PLoS ONE, 14, e0214409.

Pritchard, J. K., Stephens, M., \& Donnelly, P. (2000). Inference of population structure using multilocus genotype data. Genetics, 155, 945-959. 
R Core Team. (2020). R: A language and environment for statistical computing. R Foundation for Statistical Computing, Vienna, Austria. https://www.R-project.org.

Ramasamy, R. K., Ramasamy, S., Bindroo, B. B., \& Naik, V. G. (2014). STRUCTURE PLOT: a program for drawing elegant STRUCTURE bar plots in user friendly interface. SpringerPlus, 3: 431.

Ramsay, L., Koh, C., Konkin, D., Cook, D., Penmetsa, V., Dongying, G., Coyne, C., Humann, J., Kaur, S., Dolezel, J., Bett, K. E. (2019). Lens culinaris CDC Redberry Genome Assembly v2.0. Retrieved from https://knowpulse.usask.ca/genome-assembly/Lcu.2RBY

Sari, E., Bhadauria, V., Ramsay, L., Borhan, M. H., Lichtenzveig, J., Bett, K. E., ... Banniza, S. (2018). Defense responses of lentil (Lens culinaris) genotypes carrying non-allelic ascochyta blight resistance genes to Ascochyta lentis infection. PLoS ONE, 13, e0204124.

SAS Institute. (2015). The SAS System for Windows. Version 9.3. Cary, NC: SAS Institue, Inc. Knapp, S. J., Stroup, W. W., \& Ross, W. M. (1985). Exact confidence intervals for heritability on a progeny mean basis. Crop Science, 25, 192-194.

Sekhwal, M. K., Li, P., Lam, I., Wang, X., Cloutier, S., \& You, F. M. (2015). Disease resistance gene analogs (RGAs) in plants. International Journal of Molecular Sciences, 16, 1924819290.

Shaikh, R., Diederichsen, A., Harrington, M., Adam, J., Conner, R. L, \& Buchwaldt, L. (2013). New sources of resistance to Colletotrichum truncatum race $\mathrm{Ct} 0$ and $\mathrm{Ct} 1 \mathrm{in}$ Lens culinaris Medikus subsp. culinaris obtained by single plant selection in germplasm accessions. Genetic Resources and Crop Evolution, 60, 193-201.

Silva, D. N., Talhinhas, P., Cai, L., Manuel, L., Gichuru, E. K., Loureyro, A., ... Batista, D. (2012). Host-jump drives rapid and recent ecological speciation of the emergent fungal pathogen Colletotrichum kahawae. Molecular Ecology, 21, 2655-2670.

Slinkard, A. E. (1981). Eston lentil. Canadian Journal of Plant Science, 61, 733-734.

Sonnante, G., Hammer, K., \& Pignone, D. (2009). From the cradle of agriculture a handful of lentils: History of domestication. Rendiconti Lincei, 20, 21-37.

Srivastava, R. P., \& Vasishtha, H. (2012). Saponins and lectins of Indian chickpeas (Cicer arietinum) and lentils (Lens culinaris). Indian Journal of Agricultural Biochemistry, 25, 44-47.

Subedi, M., Bett, K. E., Khazaei, H., \& Vandenberg, A. (2018). Genetic Mapping of Milling Quality Traits in Lentil (Lens culinaris Medik.). Plant Genome, 11, 1-10.

Tanksley, S., \& McCouch, S. (1997). Seed banks and molecular maps: unlocking genetic potential from the wild. Science, 277, 1063-1066.

Tar'an, B., Buchwaldt, L., Tullu, A., Banniza, S., Warkentin, T. D., \& Vandenberg, A. (2003). Using molecular markers to pyramid genes for resistance to ascochyta blight and anthracnose in lentil (Lens culinaris Medik.). Euphytica, 134, 223-230.

Tullu, A., Banniza, S., Tar'an, B., Warkentin, T., \& Vandenberg, A. (2010). Sources of resistance to ascochyta blight in wild species of lentil (Lens culinaris Medik.). Genetic Resources and Crop Evolution, 57, 1053-1063. 
Tullu, A., Buchwaldt, L., Lulsdorf, M., Banniza, S., Barlow, B., Slinkard, A. E., ... Vandenberg, A. (2006). Sources of resistance to anthracnose (Colletotrichum truncatum) in wild Lens Species. Genetic Resources and Crop Evolution, 53, 111-119.

Tullu, A., Buchwaldt, L., Warkentin, T., Tar'an, B., \& Vandenberg, A. (2003). Genetics of resistance to anthracnose and identification of AFLP and RAPD markers linked to the resistance gene in PI 320937 germplasm of lentil (Lens culinaris Medikus). Theoretical and Applied Genetics, 106, 428-234.

Turner, S. D. (2014). qqman: An R package for visualizing GWAS results using Q-Q and Manhattan plots. bioRxiv, https://www.biorxiv.org/content/early/2014/05/14/005165.

Vandenberg, A., Banniza, S., Warkentin, T. D., Ife, S., Barlow, B., McHale, S., ... Slinkard, A. E. (2006). Cultivar description CDC Redberry lentil. Canadian Journal of Plant Science, 86: 497- 498.

Vandenberg, A., Kiehn, F., Vera, C., Gaudiel, R., Buchwaldt, L., Dueck, S., ... Slinkard, A. E. (2002). CDC Robin lentil. Canadian Journal of Plant Science, 82, 111-112.

Wu, Y., Bhat, P. R., Close, T. J., \& Lonardi, S. (2008). Efficient and Accurate Construction of Genetic Linkage Maps from the Minimum Spanning Tree of a Graph. PLoS Genetics, 4, e1000212.

Wright, D. M, Neupane, S., Heidecker, T., Haile, T. A., Chan, C., Coyne, C. J., ... Bett, K. E. (2021). Understanding photothermal interactions will help expand production range and increase genetic diversity of lentil (Lens culinaris Medik.). Plants, People, Planet, 3, 171181.

Xiao, S., Emerson, B., Ratanasut, K., Patrick, E., O’Neill, C., Bancroft, I., \& Turner, J.G. (2004). Origin and maintenance of a broad-spectrum disease resistance locus in Arabidopsis. Molecular Biology and Evolution. 21, 1661-1672.

Yano, K., Yamamoto, E., Aya, K., Takeuchi, H., Lo, P-C., Hu, L., ... Matsuoka, M. (2016). Genome-wide association study using whole-genome sequencing rapidly identifies new genes influencing agronomic traits in rice. Nature Genetics, 48, 927-34.

Zhang, Z., Ersoz, E., Lai, C., Todhunter, R. J., Tiwari, H., Gore, M. A, .. Buckler, E. S. (2010). Mixed linear model approach adapted for genome-wide association studies. Nature Genetics, 42, 355-360.

Zheng, X., Levine, D., Shen, J., Gogarten, S. M., Laurie, C., \& Weir, B. S. (2012). A Highperformance Computing Toolset for Relatedness and Principal Component Analysis of SNP Data. Bioinformatics, 28, 3326-3328.

Zhu, C., Gore, M., Buckler, E. S., \& Yu, J. (2008). Status and Prospects of Association Mapping in Plants. Plant Genome, 1, 5-20. 